\title{
THE PHYSICAL PROPERTIES OF CEMENTITIOUS COMPOSITES FOR APPLICATIONS IN A HIGH-TEMPERATURE ENERGY STORAGE DEVICE
}

\author{
Stanislav ŠŤastník*, Lenka BodnÁrová \\ Brno University of Technology, Faculty of Civil Engineering, Veveři 95, 602 00 Brno, Czech Republic \\ * corresponding author: stastnik.s@fce.vutbr.cz
}

\begin{abstract}
The article was focused on experimental verification of the identification of thermal characteristics of high-performance cementitious composites at elevated temperatures. The purpose of the research was to verify the properties, and consequently the suitability of these special cement composites for the production of a heat storage device for the long-term accumulation of thermal energy. The theoretical prerequisites for the identification of thermal characteristics and the practical procedure of experimental verification of the characteristics were presented. A modified procedure for the determination of temperature conductivity $\alpha$ by the hot wire method was suggested. Subsequently, the experimental verification and the results of the measured thermal characteristics were listed - and the temperature conductivity $\alpha$, the thermal conductivity $\lambda[\mathrm{w} / \mathrm{m} \cdot \mathrm{K}]$, the volumetric heat capacity and the specific heat capacity $c[\mathrm{~J} / \mathrm{kg} \cdot \mathrm{K}]$ by the modified method were determined.
\end{abstract}

KEYwords: Cementitious composite, thermal conductivity, volume heat capacity, specific heat capacity, carbon nanotube.

\section{INTRODUCTION}

Continuous growth in greenhouse gas emissions and fuel price increases are key drivers for the more efficient use of renewable energy sources. Renewable sources have a practically limitless lifespan as they consist of solar energy, wind energy, geothermal energy, hydropower and tidal energy. The most effective source of energy is direct sunlight, and solar energy is used by photovoltaic power plants. The current problem is the realisation of the solar energy collection and its storage in a heat storage device for long-term accumulation of thermal energy. At present, the use of renewable energy sources is realised only in so-called island systems.

The proposed heat storage system consists of a high-performance cementitious composite heat storage device and a thermal insulating cover. The aim of this system is to accumulate and maintain the temperature range within the required limits, so that it depends upon its thermal accumulation characteristics. These thermal accumulation characteristics are determined by physical thermal characteristics including, in particular, the specific heat capacity and thermal conductivity. An important role in terms of heat accumulation is also played by the bulk density.

The working temperature of the solar accumulating storage device ranges from $250^{\circ} \mathrm{C}$ to $800^{\circ} \mathrm{C}$. The temperature level is dependent not only on the supply and pumping of energy, but on the insulation of the accumulating storage device. For proper functioning and long-term durability, the choice of a suitable material for the accumulating storage device is crucial. One option is to construct an accumulating storage device from concrete. Resistance during exposure to high temperatures is very important. Another valid aspect is thermal conductivity. The higher the thermal conductivity of the storage device material, the faster the accumulation progress. The addition of carbon nanoparticles can positively affect the durability of the cement composites as well as the thermal conductivity of the composites. For example, $1-3$ lists the results of the thermal conductivity measurement of cementitious nanocomposites with carbon nanotubes, and [4] lists the changes in thermal conductivity of polymer matrix composites. These new findings have been taken into account when designing the structure of experimentally tested high-performance cementitious composites. Within the research work, the requirements for the thermal characteristics of the cement composite were defined. Subsequent experimental works consisted in identifying the thermal characteristics of selected cementitious composites at elevated temperatures.

\section{PREREQUisites FOR IDENTIFying THERMAL CHARACTERISTICS}

In order to estimate the effects of heat-stressed building structures, it is necessary to know the values of the basic thermal characteristics of the materials used. The classical evolutionary Fourier equation represents non-stationary heat transfer. To study the temperature distribution of a given structure, it is necessary to know the thermal conductivity values, $\lambda$, if the process is stationary. It is also necessary to know the specific heat capacity $c$ related to the density of the material $\rho$. The formulation of the Fourier equation 
in Cartesian coordinates is as follows:

$$
\frac{\partial T}{\partial t}=\frac{\lambda}{\rho \cdot c}\left(\frac{\partial^{2} T}{\partial x^{2}}+\frac{\partial^{2} T}{\partial y^{2}}+\frac{\partial^{2} T}{\partial z^{2}}\right)+\frac{Q_{z}}{\rho \cdot c}
$$

After transforming the relation (1) into the cylindrical coordinates in the two-dimensional space, we obtain an equation:

$$
\frac{d T}{d t}=\alpha\left(\frac{\partial^{2} T}{\partial r^{2}}+\frac{1}{r} \frac{\partial T}{\partial r}+\frac{1}{r^{2}} \frac{\partial^{2} T}{\partial \varphi^{2}}\right)+\frac{Q_{z}}{\rho \cdot c}
$$

A suitable method for measuring refractories in the temperature range from $20^{\circ} \mathrm{C}$ to $800^{\circ} \mathrm{C}$ is the hot wire method. This method allows computational identification of thermal conductivity and heat capacity at elevated temperatures. Material identification by the heating wire method uses the following assumptions:

(1.) The heating wire is considered to be thin and its thermal and temperature conductivity can be neglected;

(2.) The material sample can be assumed to be a long rotary cylinder with a large radius $r$, in whose axis lies the heating wire;

(3.) The heating wire, at the time $t=0$, supplies the constant heat output $Q_{w}\left[\mathrm{~W} \cdot \mathrm{m}^{-1}\right]$ to the unit of length, so that the power $Q_{w l}[\mathrm{~W}]$ can be related to the surface area of the cylinder with content $2 \pi r l$, where $r$ is the distance of all points from the heating wire with consideration of the previous assumptions for the limit transition $r \rightarrow 0_{+}$;

(4.) The material is considered homogeneous and isotropic with the scalar characteristics of $\lambda$, and $\alpha$;

(5.) The characteristics $\lambda$ and $\alpha$ can be at a given temperature level and can be considered as independent of the temperature of the sample $T(r, t)$ for any radius $r \in R_{+}$and time (the set $R_{+}$is considered as a set of positive real numbers);

(6.) The ambient temperature of the sample is unchanged during the measurement.

Under these assumptions, it is possible to use a classical analytical relation (2) for the distribution of temperature in the measured sample 5. As a result, the ambient temperature of the heating wire at the distance $r$ in time $t$ reaches the following values:

$$
T(r, t)=T_{0}+\frac{Q}{4 \pi \lambda} E i\left(\frac{r^{2}}{4 \alpha t}\right) \forall(r, t) \in R_{+} \times R_{+}
$$

in which $E i$ represents an exponential integral

$$
\operatorname{Ei}(\beta)=\int_{\beta}^{\infty} \frac{\exp (-u)}{u} d u
$$

$E i$ is defined for any number $\beta \in R_{+}$. Therefore, in accordance with (3), the following equation is applicable:

$$
\begin{array}{r}
T(r, t)=T\left(r, t_{a}\right)-\frac{Q}{4 \pi \lambda}\left(E i\left(\frac{r^{2}}{4 \alpha t}\right)-E i\left(\frac{r^{2}}{4 \alpha t_{a}}\right)\right) \\
\forall(r, t) \in R_{+} \times R_{+} \quad(5)
\end{array}
$$

It is obvious that the temperature $T(r, t)$ from equation (5) conforms to the evolutionary partial heat equation without internal sources for the axially symmetrical task given by the correlated relation

$$
r \kappa \frac{\partial T}{\partial t}=\frac{\partial}{\partial r}\left(\lambda \frac{\partial T(r, t)}{\partial r}\right) \forall(r, t) \in R_{+} \times R_{+}
$$

with the initial condition at time $t \rightarrow 0_{+}$

$$
\lim _{t \rightarrow 0_{+}} T(r, t)=T_{0} \quad \forall r \in R_{+}
$$

the boundary condition of the Dirichlet type

$$
\lim _{r \rightarrow \infty} T(r, t)=T_{0} \quad \forall t \in R_{+}
$$

and the Neumann type boundary condition

$$
\lim _{r \rightarrow 0_{+}} \frac{-\lambda \frac{\partial T(r, t)}{\partial r}}{\frac{Q}{2 \pi r}}=1 \quad \forall t \in R_{+}
$$

In condition (9), the denominator is the Fourier heat flux. The derivation of equation 10 from the standard heat distribution equation in Cartesian coordinates is conducted in [6] Chapter 9. The author also discussed this issue in [7] Chapter 3.

The thermal conductivity $\lambda$ represents the density of the heat flow divided by the temperature gradient. The derived physical quantity is the thermal conductivity $\alpha$, which is formulated by relation $\alpha=\frac{\lambda}{\kappa}$.

\section{Determination of thermal CONDUCTIVITY BY THE METHOD OF HEATING WIRE (ISO 8894-1)}

Experimental determination of thermal conductivity is carried out according to ISO 8894-1 8, because the procedure allows the determination of the thermal conductivity coefficient $\lambda$ by the dynamic method for dense materials, with a thermal conductivity of up to $25 \mathrm{~W} \cdot \mathrm{m}^{-1} \cdot \mathrm{K}^{-1}$. The temperature rise time of the heating wire is measured as a result of the propagation of the thermal wave induced by the supplied constant power through the line conductor, which is stored inside the monitored sample of the building material. The sample is placed in a heated space, whereby the condition for the evaluation of the physical quantities is the stability of the ambient temperature at a tolerance of $10 \mathrm{~K}$.

The heating wire is made of a material called kanthal, with a length equal to the length of the test specimen, and the ends of the heating wire are connected to a supply voltage and a digital multimeter. A stabilised power supply, whose voltage and current supply the corresponding heat output to the heating wire, may not fluctuate by more than $2 \%$ during the measurement. The required heat capacity of the heating wire must be greater than $250 \mathrm{~W} \cdot \mathrm{m}^{-1}$. The measuring thermocouples are of a $\mathrm{Ni} / \mathrm{NiCr}$ design.

Test procedure: The heating wire and measuring thermocouples are placed between the upper and lower 
part of the test body, and thermocouple 2 is placed in a groove in the centre of the contact edge of the fitting before eventually being sealed. Thermocouple 1 is placed into the groove in the test material perpendicular to the heating wire. The test unit is then placed in the furnace. Due to the uniform heating in the furnace, the test unit is placed on mats made of a similar material to the test sample. The heating wire is connected to a stabilised DC source.

If the conditions given by the relevant standard (ISO 8894-1, see point a) to f)) are met, it is possible to connect the current from the stabilised source and gradually, at certain time intervals, to measure temperatures on both thermocouples. The effect of the heating power must be carried out during the time $\mathrm{t}$ determined for the assumed $\lambda, \rho$ and $c$ values according to ISO 8894-1. After this time, the heating current is interrupted and the recording of temperature course is terminated. Once the temperature between the two thermocouples has stabilised, the measurement can be repeated under the same conditions. [8]

The evaluation of the results is taken from the sequence of temperature difference quotients at the current point in time $t_{i}$ and its double. The temperature differences are calculated to the start-up temperature of the power supply. Thermal conductivity is calculated based on the following relationship:

$$
\begin{gathered}
\lambda=\frac{P}{4 \pi L} \frac{-E i\left(\frac{-r^{2}}{4 \alpha t}\right)}{\Delta \theta(t)} \\
P=U \cdot I
\end{gathered}
$$

In the above relation, $E i$ is the exponential integral given by the dependence (9) in which the expression is formulated as follows:

$$
u=\frac{-r^{2}}{4 \alpha t}
$$

The measured temperatures are tabulated at the same time intervals. When evaluating the results, the calculation is performed by using the ratio of differences of the measured temperatures at time $t$ and also at double time $2 t$. The ratio of the measured differences is designated by the symbol $Z$ :

$$
z=\frac{\Delta \theta(2 t)}{\Delta \theta(t)}
$$

The value of the exponential integral $E i$ is a function of this variable and is tabulated in ISO 8894-1 8. In the measurement table, the $Z$ values are calculated for each time point to which the corresponding integral is assigned. In each $i$-line, according to relation (16), the thermal conductivity $\lambda_{i}$ is calculated. The resulting thermal conductivity is evaluated as the average of all $\lambda_{i}$.

$$
\lambda=\frac{\sum_{i=1}^{n} \lambda_{i}}{n}
$$

Due to the fact that the number of measured values, and therefore, the length of measuring time intervals are limited because of the calculation slowness, the accuracy of the evaluation is reduced.

\section{EXPERIMENTAL PART}

\subsection{Procedure For Determining the THERMAL CONDUCTIVITY AND HEAT CAPACITY OF THE MATERIAL BY A MODIFIED HOT WIRE METHOD}

Suppose we have now experimentally measured $T^{*}(r, t)$ and $T(r, t)$ temperature values expressed analytically on the set $R_{+} \times R_{+}$. We now introduce the function $F(\lambda, \kappa)$ so that the following is valid:

$$
F(\lambda, \kappa)=\frac{1}{2}\left(T-T^{*}, T-T^{*}\right)
$$

The formula in brackets (.,.) denotes the scalar product in the Lebesgue space of functions integrable in the square on the set $R_{+} \times R_{+}$with a suitably chosen weight $w(r, t)$. The minimum function (15) clearly corresponds to the $\kappa$ and $\lambda$ values. If we specifically know the temperature values for radii $n\left\{r_{1} \ldots r_{n}\right\}$ and times $m\left\{t_{1} \ldots t_{m}\right\}$, the mentioned integral scalar product for any admissible functions $\varphi$ and $\psi$ can be defined by the relation:

$$
(\varphi, \psi)=\iint_{0}^{\infty} w(r, t) \varphi(r, t) \psi(r, t) d r d t
$$

for positive discrete weight $w_{i j}$ properties:

$$
\sum_{i=1}^{n} \sum_{j=1}^{m} w_{i j}=1
$$

And the Dirac two-dimensional measure:

$$
w(r, t)=\sum_{i=1}^{n} \sum_{j=1}^{m} \delta\left(r-r_{i}, t-t_{j}\right) \cdot w_{i j}
$$

then $F$ is generally a nonlinear function of two variables $\kappa$ and $\lambda$. In the calculation $15 n=1$ and $w_{11}=w_{12}=\ldots w_{1 m}=1 / m$ are selected. If, in the definition (15), a variable $\alpha=Q / 4 \pi \lambda$ is considered instead of the variable $\lambda$, then it is based on $(18):+$

$$
\left(\ln \frac{t}{t_{a}}, \ln \frac{t}{t_{a}}\right) \alpha=\frac{1}{2}\left(T^{*}-T_{a}^{*}, \ln \frac{t}{t_{a}}\right)
$$

The values with the asterisk in the formula 19 represent experimentally measured data. From this, it is possible to directly calculate the thermal conductivity value $\alpha$. For simplicity, let us consider that we want to determine the value $\alpha$ from another independent experiment. From the previous, it differs only by measuring it at a different distance from the line power source, so $r_{1}=d$. Instead of variable a, we can now 
consider function $F$ as a function of the new variable $b=\frac{r_{1}^{2}}{4 \kappa}$. Then equation (5) has the following form:

$$
\begin{array}{r}
T\left(r_{1}, t\right)=T\left(r_{1}, t_{a}\right)+\kappa\left(E i\left(\frac{b}{t}\right)-E i\left(\frac{b}{t_{a}}\right)\right) \\
\forall t \in R_{+}
\end{array}
$$

If we mark the first and second derivatives of the function $F$ with apostrophe, then we gradually get the values necessary for the subsequent calculations. The basic function $F$ has the following form:

$$
F(\lambda, b)=\frac{1}{2}\left(T-T^{*}, T-T^{*}\right)
$$

The first derivation:

$$
F^{\prime}=\left(T^{\prime}, T-T^{*}\right)
$$

The second derivation:

$$
F^{\prime \prime}=\left(T^{\prime}, T^{\prime}\right)+\left(T^{\prime \prime}, T-T^{*}\right)
$$

If the first and second derivations of function (15) are known, then it is possible to apply the iterative Newton method of tangents in the form:

$$
F^{\prime \prime}\left(b-b^{\sim}\right)=F^{\prime}
$$

The wave line above variable $b$ indicates a refined parameter value. Generalising this approach is therefore the choice $n=2$ with the condition of approaching the distance $r_{1} \rightarrow 0$ from the right and $r_{2}=d$.

Additionally, the procedure may be modified by the Levenberg-Marquardt technique according to [6] in Chapter 10b. If we want to formally observe the standardised method according to ISO 8894-1, we can evaluate the thermal conductivity for $r_{1} \rightarrow 0_{+}$, where it is not necessary to know the $\kappa$ value. It would then be possible to indirectly detect the value $b$ by the iterative process and afterwards set parameter $\kappa$ from this value. In the research work, the calculation of $\lambda$ and $\kappa$ is verified by experiment, which applies the modified heating wire method.

The difference between this method and the classical method according to ISO 8894 is, on the one hand, in the method of supplying heat energy through the power that is realised by the heating wire and then the subsequent cooling of the sample after the interruption of the supply; and on the other hand, in the distribution of thermocouples for measuring the temperature of the gradual thermal wave. The process is time-limited by both heating and cooling time (both time intervals are the same).

\subsection{ARrangement of An EXPERIMENTAL ASSEMBLY FOR THE PROCEDURE OF DETERMINING THE THERMAL CONDUCTIVITY BY THE HOT WIRE METHOD}

The arrangement of samples and thermocouples for the experiment differed from the arrangement for measurement by the $\lambda$ method according to ISO 8894-1 [8].

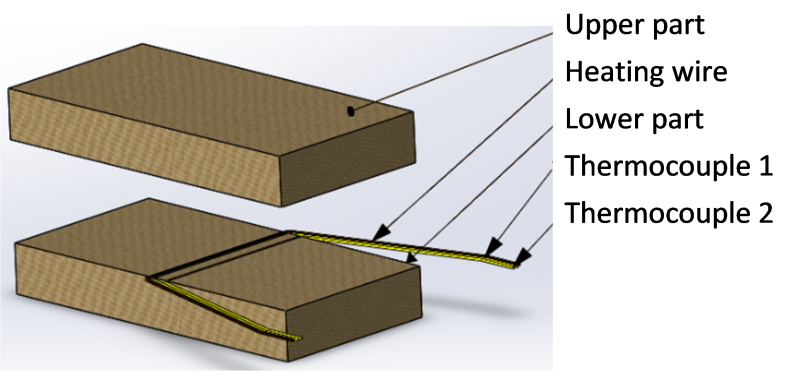

Figure 1. The sample arrangement when determining the thermal conductivity $\lambda$ and thermal capacity $c$ by the Hot wire method.

The first measuring thermocouple lie in close proximity to the heating wire, usually at a distance of $3 \mathrm{~mm}$, and the other thermocouple lies parallel to the heating wire at a distance of $15 \mathrm{~mm}$. The schematic arrangement of the measured block is shown in Figure 1. The process of the temperature and measurement setting is controlled by the computer.

\section{Results AND Discussion}

\subsection{Summary OF KNOWLEDGE ABOUT MATERIALS FOR REALISATION OF SENSIBLE STORAGE DEVICES}

The objective of the experiment was to prove the properties of silicate materials in terms of their use in the realisation of sensible high-capacity and hightemperature storage containers. For this purpose, samples of cementitious composites resistant to temperatures above $800{ }^{\circ} \mathrm{C}$ were selected for their realisation. Indicators of the suitability for use of the chosen materials were the physical values of the thermal conductivity $\lambda$ and the specific heat capacity $c$.

A suitable storage container must have such properties to ensure the required energy use. The storage container is charged by insolation of the collector during the climatic year.

A validated sensible energy storage device can be used as a power supply for a family home and has been designed for minimal temperature loss.

The input data were density, specific heat capacity, immediate power output over a one-year period, storage container size and daily consumption. The capacity of the storage container and its dynamic behaviour depends, inter alia, mainly on the value of the specific heat capacity of the building material and its density. Another important factor of energy accumulation is its thermal conductivity. The higher the thermal conductivity of the container's building material, the faster the accumulation progress. The working temperatures of the storage container vary between $200{ }^{\circ} \mathrm{C}$ and $500{ }^{\circ} \mathrm{C}$. In this area, we have to look for values with a favourable specific heat capacity and thermal conductivity.

Cementitious composites of various compositions were tested. Five variants of cement composites (CA1, CA2, CC1, CC2, CQ) were tested. The matrix of 


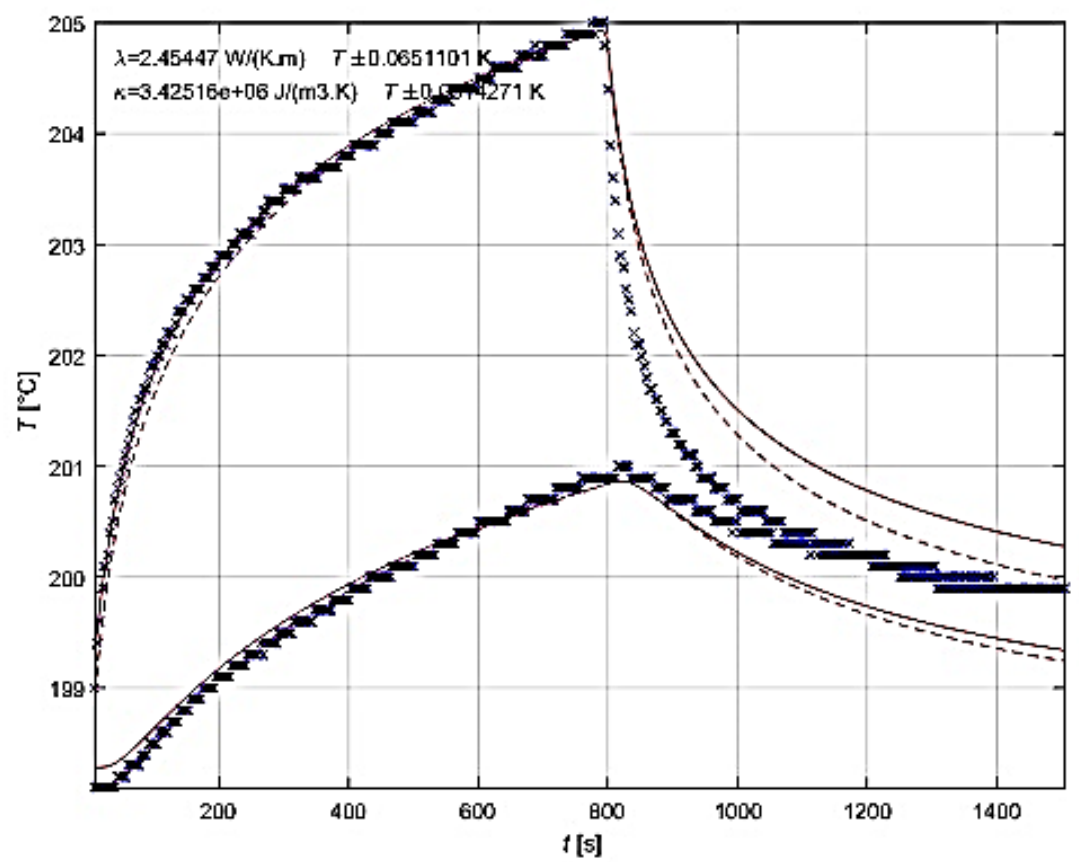

Figure 2. Temperature changes $T\left[{ }^{\circ} \mathrm{C}\right]$ in the sample of cementitious composite $\mathrm{CC} 1$ at $200{ }^{\circ} \mathrm{C}$ depending on time $t[\mathrm{~s}]$.

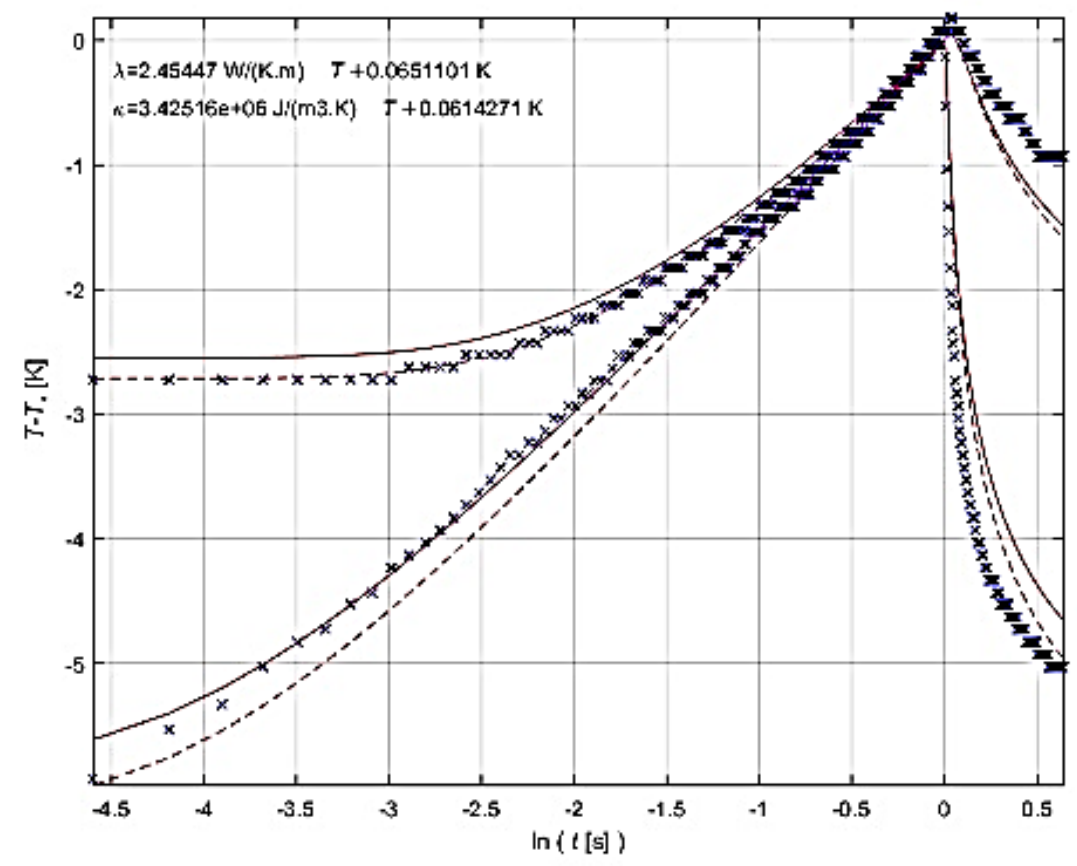

FiguRE 3 . The iterative convergence process of $\lambda$ and $\kappa$.

the CA1 and CA2 composites was made from aluminous cement, composite matrix CC1, CC2 was based on Portland cement and sample CQ was magnesium based composite.

Figure 2 shows the change of temperatures $T$ in the sample of the cementitious composite at $200{ }^{\circ} \mathrm{C}$ with respect to time $t$, and Figure 3 shows the convergence of iterations $\lambda$ and $\kappa$ for the sample of the cementitious composite.

The measurement results of the selected cementitious composites are presented in Charts 4 and 5 .

The measurement results of the cementitious composites with different composition are presented on the diagrams in Figures 6 and 7 


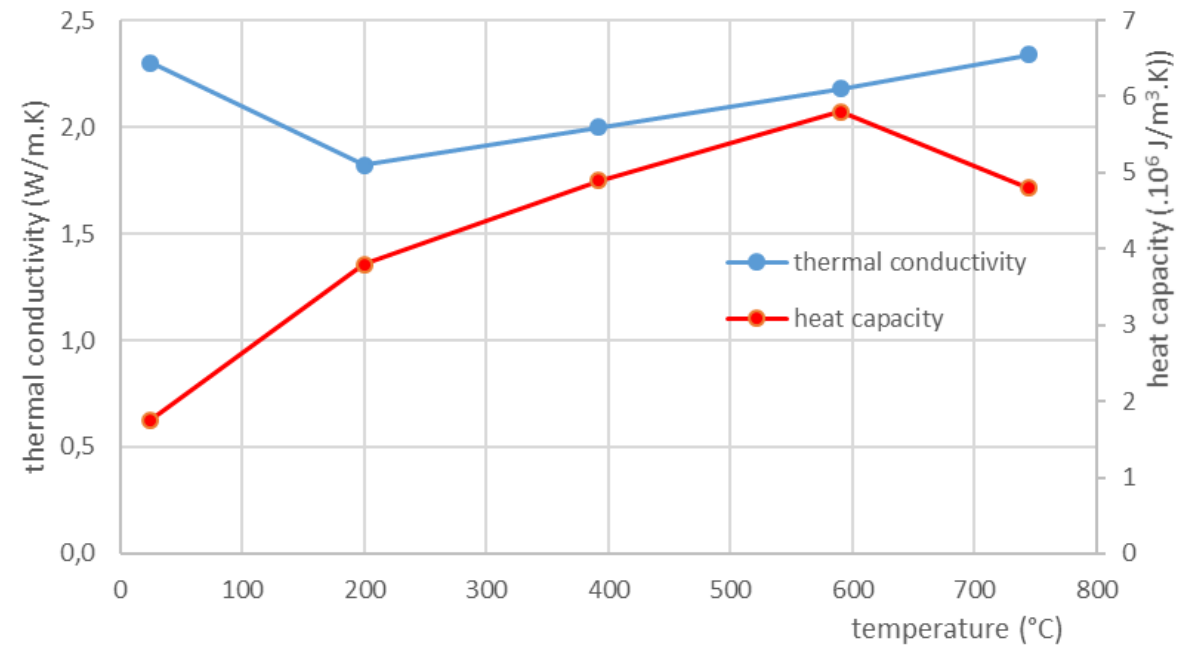

Figure 4 . The course of the change in thermal conductivity $[\mathrm{w} / \mathrm{m} \cdot \mathrm{K}]$ and volume heat capacity $\left[\mathrm{J} / \mathrm{m}^{3} \cdot \mathrm{K}\right]$ at the temperature for cementitious composite $\mathrm{CC} 1$ depending on ambient temperature $\left[{ }^{\circ} \mathrm{C}\right]$.

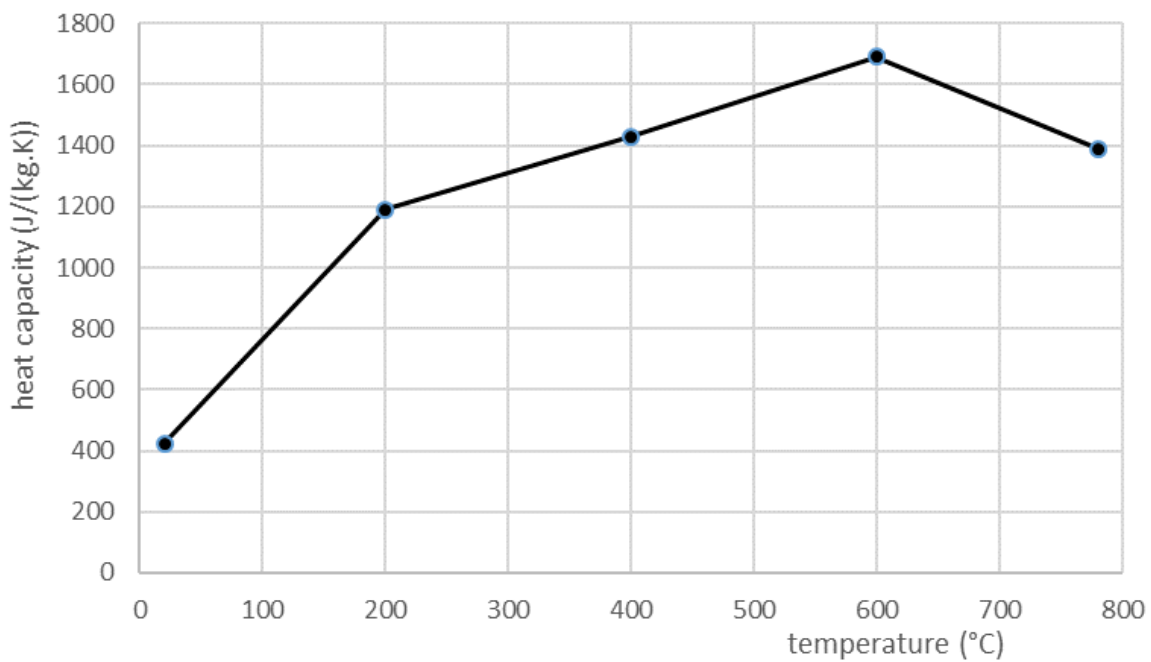

FiguRE 5. Change in specific heat capacity $c[\mathrm{~J} / \mathrm{kg} c \operatorname{dot} \mathrm{K}]$ for cementitious composite $\mathrm{CC} 1$ depending on ambient temperature $\left[{ }^{\circ} \mathrm{C}\right]$.

\section{Conclusion}

For the intended application of the high-performance cementitious composite for the heat accumulating storage device, it is necessary to achieve the highest possible thermal conductivity and heat capacity. The thermal conductivity value is related to the rate of equalisation of the temperature differences in the storage device volume, which is the decisive parameter for charging and discharging of the high-temperature heat storage device. The heat capacity value of the storage device core material determines the total heat content, and is a decisive parameter in determining its geometric volume. Thermal identification over a wide range of operating temperatures is necessary to verify the thermal behaviour of the various cementitious composites under the real conditions of incorporation into the core of the high-temperature heat storage device.
The best results were obtained with materials with a high magnesium oxide content. These results, however, are not presented in the comparisons in Figures 6 and 7 because this material is incomparable, in terms of price, with the tested high-performance cementitious composites, and its price does not currently allow its incorporation into the real-world construction of the high-temperature heat storage device.

By comparing the thermal conductivity and thermal capacity values in the temperature range of the operating temperatures of $20^{\circ} \mathrm{C}$ to $800^{\circ} \mathrm{C}$, it appears to be the most suitable cementitious composite with the designation CC1, whose favourable physical properties are also related to its density.

The useful physical properties of the final sensible storage device made of CC1 material are closely related to the use of the thermal insulating layers in the packaging construction, the overall needed size of the storage device, and its operating states. The specific 


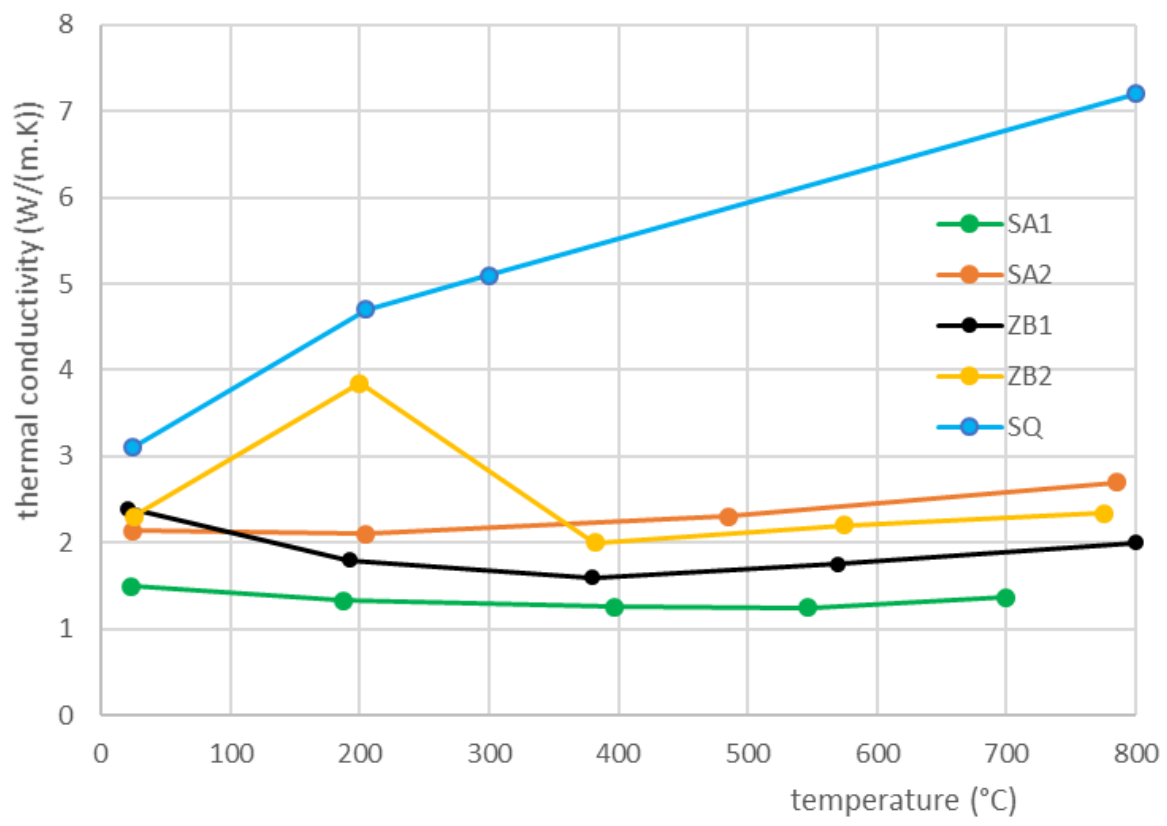

FiguRE 6. Change in thermal conductivity depending on the ambient temperature for the monitored cementitious composites.

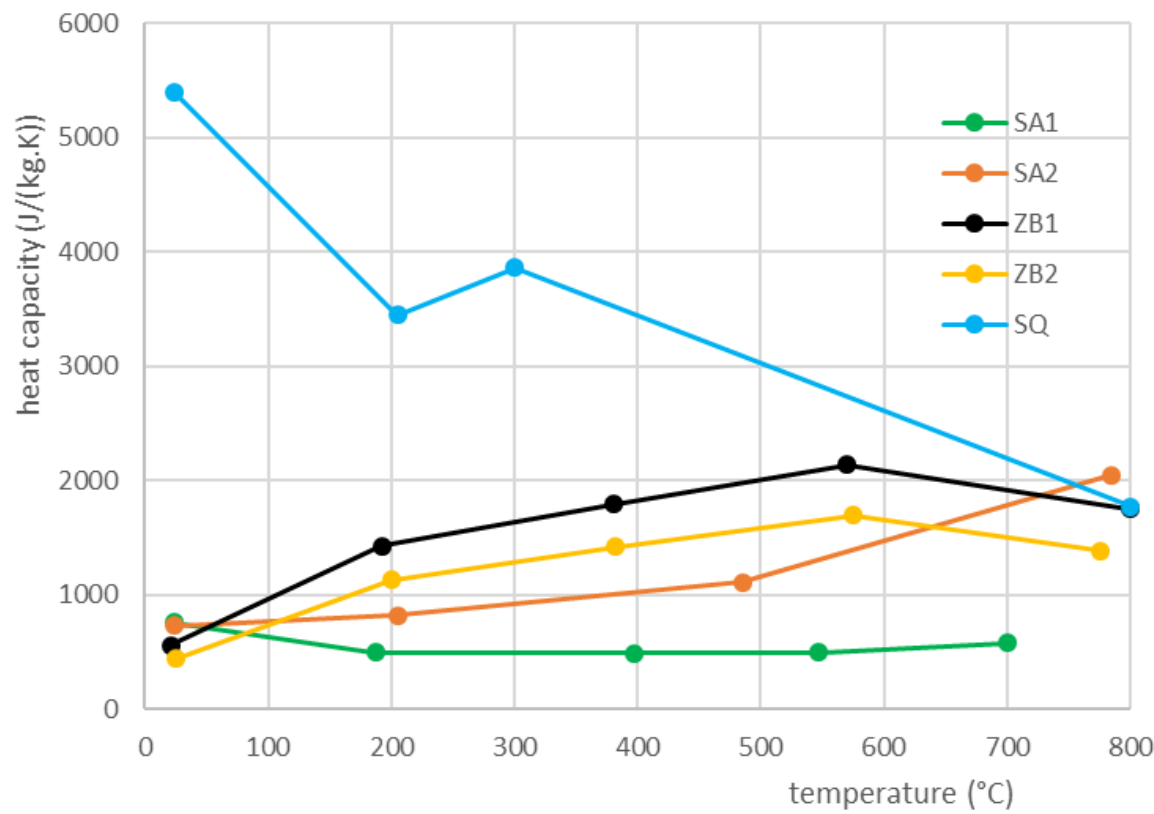

FiguRE 7. Change in heat capacity depending on the ambient temperature for monitored cementitious composites.

design and arrangement of the high-temperature heat storage device will depend on the thermal balance of the energy flows, their time distribution, etc. However, due to the favourable properties of the storage device core material, it provides the most favourable physical characteristics.

\section{ACKNOWLEDGEMENTS}

This outcome has been achieved with the financial support of Project FAST-S-18-5410, supported by Brno University of Technology.

\section{REFERENCES}

[1] M. Hassanzadeh-Aghdam, R. Ansari, M. Mahmoodi, et al. A comprehensive study on thermal conductivities of wavy carbon nanotube-reinforced cementitious nanocomposites. Cement and Concrete Composites 90:108-118, 2018.

DOI:10.1016/j.cemconcomp.2017.09.021

[2] D. Exarchos, P. Dalla, I. Tragazikis, T. Matikas. The effect of CNTs reinforcement on thermal and electrical properties of cement-based materials. In Smart Sensor Phenomena, Technology, Networks, and Systems Integration 2015, vol. 9436, pp. 94360E-1. 2015. 
[3] A. L. Pisello, A. D'Alessandro, S. Sambuco, et al. Multipurpose experimental characterization of smart nanocomposite cement-based materials for thermalenergy efficiency and strain-sensing capability. Solar Energy Materials and Solar Cells 161:77-88, 2017.

[4] C. Huang, X. Qian, R. Yang. Thermal conductivity of polymers and polymer nanocomposites. Materials Science and Engineering R-Reports 132:1-22, 2018. DOI:10.1016/j.mser.2018.06.002.

[5] H. S. Carslaw, J. C. Jaeger. Conduction of Heat in Solids. Clarendon Press Oxford, 1959.
[6] P. Bochev, M. Gunzburger. Least-Squares Finite Element Methods. Springer, New York, 2009.

[7] L. Dohnalová, I. Trhlíková, O. Zmeškal. Temperature dependence of thermal parameters of building materials. In Proceedings of Thermophysics in Podkylava, pp. 194-207. Bratislava, 2013.

[8] ISO 8894-1 Refractory materials - Determination of thermal conductivity - Part 1: Hot-wire methods (cross-array and resistance thermometer). Standard, European Committee for Standardization, 2011. 